Journal of the Operations Research

Society of Japan

Vol. 44, No. 1, March 2001

\title{
A DISCRETE BASS MODEL AND ITS PARAMETER ESTIMATION
}

\author{
Daisuke Satoh \\ NTT Service Integration Laboratories
}

(Received November 19, 1998; Final May 8, 2000)

\begin{abstract}
A discrete Bass model, which is a discrete analog of the Bass model, is proposed. This discrete Bass model is defined as a difference equation that has an exact solution. The difference equation and the solution respectively tend to the differential equation which the Bass model is defined as and the solution when the time interval tends to zero. The discrete Bass model conserves the characteristics of the Bass model because the difference equation has an exact solution. Therefore, the discrete Bass model enables us to forecast the innovation diffusion of products and services without a continuous-time Bass model.

The parameter estimations of the discrete Bass model are very simple and precise. The difference equation itself can be used for the ordinary least squares procedure. Parameter estimation using the ordinary least squares procedure is equal to that using the nonlinear least squares procedure in the discrete Bass model.

The ordinary least squares procedures in the discrete Bass model overcome the three shortcomings of the ordinary least squares procedure in the continuous Bass model: the time-interval bias, standard error, and multicollinearity.
\end{abstract}

\section{Introduction}

Since its introduction to marketing in the 1960 s $[1,2,7,11,19,22]$, the diffusion theory perspective has been of interest to scholars of consumer behavior, marketing management, and management and marketing science. The main impetus underlying the work done in this area is a new-product growth model developed by Bass [2].

The Bass model has been investigated in mainly three aspects: adopter categorization $[14,25]$, the communication structure between the two assumed groups of adopters of 'innovators' and 'imitators' [24], and the development of diffusion models by specifying adoption decisions at the individual level $[5,18]$. The Bass model and its revised forms have been successfully demonstrated for forecasting innovation diffusion in many products and services.

Bernhardt and MacKenzie [3], however, have stated that although the simple diffusion models work well in some cases, in other cases the results are poor. They suggest that the success of diffusion models has been due to a "judicious choice of situation, population, innovation and time frame for evaluating the data." Heeler and Hustad [8] have reported examples of new product diffusion in an international setting where the Bass model does not perform well.

Mahajan and Wind [16] suggested that one possible reason why diffusion models work in some cases but do not perform well in others could be the particular estimation procedure used to estimate the parameters of the diffusion models. Mahajan, Srinivasan, and Mason [13] compared four estimation procedures: ordinary least squares estimation (OLS) [2], maximum likelihood estimation (MLE) [21], nonlinear least squares estimation (NLS) [10, 23], and algebraic estimation (AE) [15]. They concluded that NLS procedures provide better 
predictions and more valid estimates of standard errors for the parameter estimates than the other three estimation procedures. The NLS procedure, however, is elaborate.

Evaluation of the differential in the differential equation makes it difficult to propose a simple and accurate procedure. I do not extend the parameter-estimation procedure but propose a discrete analog of the Bass model. Hirota [9] proposed a discrete Riccati equation, which has an exact solution. The Bass model is regarded as a Riccati equation. Therefore, I derived a discrete Bass model. The result obtained by OLS is equivalent to that obtained by NLS in the parameter estimation of the discrete Bass model. NLS is the most accurate procedure and OLS is the simplest one.

\section{The Bass Model and Conventional Parameter Estimations}

Since the Bass model [2] was first reported, diffusion theory has often been used to model the first-purchase sales growth of a new product over time.

In his 1969 article, Bass suggested that the following differential equation can be used to represent the diffusion process:

$$
\frac{d N(t)}{d t}=\left(p+\frac{q}{m} N(t)\right)(m-N(t))
$$

where $N(t)$ is the cumulative number of adopters at time $t, m$ is the ceiling, $p$ is the coefficient of innovation, and $q$ is the coefficient of imitation.

Assuming $F(t)=\frac{N(t)}{m}$, where $F(t)$ is the fraction of potential adopters who adopt the product by time $t$, the Bass model can be restated as

$$
\frac{d F(t)}{d t}=(p+q F(t))(1-F(t))
$$

If $N\left(t=t_{0}=0\right)=0$, simple integration of equation (1) gives the following distribution function to represent the time-dependent aspect of the diffusion process. That is,

$$
N(t)=m\left(\frac{1-e^{-(p+q) t}}{1+\frac{q}{p} e^{-(p+q) t}}\right) .
$$

Equation (3) yields the S-shaped diffusion curve captured by the Bass model. In fact, for this curve, the point of inflection (which is the maximum penetration rate, $[d N(t) / d t]_{\max }$ ) occurs when

$$
\begin{aligned}
N\left(t^{*}\right) & =m\left(\frac{1}{2}-\frac{p}{2 q}\right), \\
t^{*} & =-\frac{1}{p+q} \log \left(\frac{p}{q}\right),
\end{aligned}
$$

and

$$
f\left(t^{*}\right)=\frac{d N\left(t^{*}\right)}{d t}=m\left(\frac{q}{4}+\frac{p}{2}+\frac{p^{2}}{4 q}\right) .
$$

Hence, if $p, q$, and $m$ are known for a particular product, equations (3)-(6) can be used to represent the product growth curve.

A number of estimation procedures have been suggested for estimating parameters $p, q$, and $m$ of the Bass model. Mahajan et al. [13] compared the four estimation proceduresthe ordinary least squares (OLS), the maximum likelihood estimation (MLE), the nonlinear 
least squares (NLS), and the algebraic estimation (AE) procedures-by applying them to several sets of data. They concluded that NLS yielded better predictions as well as more valid estimates of standard errors for the parameter estimates. On the other hand, OLS is the easiest to implement. Therefore I will explain the OLS and NLS procedures in detail in the following two sections.

\subsection{The ordinary least squares procedure}

The OLS procedure suggested by Bass [2] is one of the earliest procedures for estimating the parameters. This procedure involves estimation of the parameters by taking the discrete or regression analog of the differential equation (1). Equation (1) is discretized with an ordinary forward difference equation as follows:

$$
\begin{aligned}
N\left(t_{i}\right)-N\left(t_{i-1}\right) & =p m+(q-p) N\left(t_{i-1}\right)-\frac{q}{m} N^{2}\left(t_{i-1}\right), \\
X(i) & =\alpha_{1}+\alpha_{2} N\left(t_{i-1}\right)+\alpha_{3} N^{2}\left(t_{i-1}\right),
\end{aligned}
$$

where $\alpha_{1}=p m, \alpha_{2}=q-p$, and $\alpha_{3}=-q / m$. The data-collection interval must be constant.

Given regression coefficients ${ }^{1} \hat{\alpha}_{1}, \hat{\alpha}_{2}$, and $\hat{\alpha}_{3}$, the estimates of parameters $p, q$, and $m$ can be easily obtained as follows:

$$
\begin{aligned}
& \hat{p}=\frac{-\hat{\alpha}_{2}+\sqrt{\hat{\alpha}_{2}^{2}-4 \hat{\alpha}_{1} \hat{\alpha}_{3}}}{2}, \\
& \hat{q}=\frac{\hat{\alpha}_{2}+\sqrt{\hat{\alpha}_{2}^{2}-4 \hat{\alpha}_{1} \hat{\alpha}_{3}}}{2}, \quad \text { and } \\
& \hat{m}=\frac{-\hat{\alpha}_{2}-\sqrt{\hat{\alpha}_{2}^{2}-4 \hat{\alpha}_{1} \hat{\alpha}_{3}}}{2 \hat{\alpha}_{3}} .
\end{aligned}
$$

The main advantage of the OLS estimation procedure is that it is easy to implement. It is applicable to many diffusion models, the only exception being those models that cannot be expressed as linear in their parameters; for example, the Von Bertalanffy [4] model.

However, the OLS procedure has three shortcomings [21]. First, as is clear from equation (8), in the presence of only a few data points and the likely multicollinearity between variables $\left(N\left(t_{i-1}\right)\right.$ and $\left.N^{2}\left(t_{i-1}\right)\right)$, one may obtain parameter estimates that are unstable or possess wrong signs (see, for example, $[8,21,23]$ ). Second, the standard errors for the estimates are not available since parameters $p, q$, and $m$ are nonlinear functions of $\alpha_{1}, \alpha_{2}$, and $\alpha_{3}$. The error term, however, does contain the net effect of all sources of error. Third, the right-hand side of equation (7) will overestimate the derivative of $N(t)$ taken at $t_{i-1}$ for time intervals before the point of inflection and will underestimate after that. That is, a time-interval bias is present in the OLS approach since discrete time-series data are used to estimate a continuous-time model.

\subsection{Nonlinear least squares estimation (NLS)}

The nonlinear least squares estimation procedure suggested by Srinivasan and Mason [23] was designed to overcome some of the shortcomings of the maximum likelihood estimation procedure, which itself was designed to overcome the shortcomings of the OLS procedure of Schmittlein and Mahajan [21]. Using the cumulative distribution function given by

$$
F(t)=\frac{1-e^{-b t}}{1+a e^{-b t}}
$$

\footnotetext{
${ }^{1} \hat{\alpha}_{1}>0, \hat{\alpha}_{2}>0$, and $\hat{\alpha}_{3}<0$ because $\hat{p}, \hat{q}$, and $\hat{m}$ are positive.
} 
Srinivasan and Mason suggest that parameter estimates $\hat{p}, \hat{q}$, and $\hat{m}$ can be obtained by using the following expression for the number of adopters $X(i)$ in the $i$ th time interval $\left(t_{i-1}, t_{i}\right)$ :

$$
\begin{aligned}
& X(i)=m\left(F\left(t_{i}\right)-F\left(t_{i-1}\right)\right)+\mu_{i} ; \quad \text { or } \\
& X(i)=m\left(\frac{1-e^{-(p+q) t_{i}}}{1+(q / p) e^{-(p+q) t_{i}}}-\frac{1-e^{-(p+q) t_{i-1}}}{1+(q / p) e^{-(p+q) t_{i-1}}}\right)+\mu_{i},
\end{aligned}
$$

where $\mu_{i}$ is an additive error term. Based on equation (14), parameters $p, q$, and $m$ and their asymptotic standard errors can be directly estimated.

The nonlinear least squares estimation procedure overcomes the time-interval bias present in the OLS procedure. Furthermore, since the error term may be considered to represent the net effect of sampling errors, excluded variables (such as economic conditions and marketing mix variables), and mis-specification of the density function, the derived standard errors for the parameter estimates may be more realistic. However, since the nonlinear least squares estimation procedure employs various search routines to estimate the parameters, parameter estimates may sometimes be very slow to converge or may not converge, the final estimates may be sensitive to the starting values for $p, q$, and $m$, or the procedure may not provide a global optimum.

\section{The Discrete Bass Model}

An easy and accurate parameter estimation procedure is difficult to develop. One reason for this is that the Bass model is a continuous-time model while the data we obtain is discrete. If we had a discrete model that conserved the properties of the continuous model, the parameter estimation would likely be simpler and more accurate. I propose a discrete Bass model obtained by using a discrete Riccati equation [9]. This model is described by a difference equation. The difference equation has an exact solution, although an ordinary forward difference equation does not. The discrete Bass model enables us to forecast innovation diffusion without a continuous-time Bass model because the discrete model has an exact solution.

A Riccati equation is

$$
\frac{d u}{d t}=a(t)+2 b(t) u+c(t) u^{2}
$$

where $a(t), b(t)$, and $c(t)$ are given functions of $t$. In this paper, the Riccati equation is considered when $a, b$, and $c$ are constant. Equation (1) can be regarded as a Riccati equation by setting

$$
\begin{aligned}
& a=m p, \\
& b=\frac{q-p}{2}, \\
& c=-\frac{q}{m} .
\end{aligned}
$$

Hirota obtained a discrete Riccati equation [9] that has an exact solution. His discrete Riccati equation is described as

$$
\frac{u(t+\delta)-u(t-\delta)}{2 \delta}=a+b(u(t+\delta)+u(t-\delta))+c u(t+\delta) u(t-\delta)
$$


where $\delta$ is the constant time-difference length. The exact solution to equation (19) is given as

$$
u(t)=\frac{C_{+}+C_{-} \exp \left(\Omega\left(t-t_{0}\right)\right)}{1+\exp \left(\Omega\left(t-t_{0}\right)\right)}
$$

where

$$
\begin{aligned}
C_{ \pm} & =\frac{-b \pm \sqrt{b^{2}-a c}}{c} \\
\tanh (\delta \Omega) & =2 \delta \sqrt{b^{2}-a c}
\end{aligned}
$$

By using the discrete Riccati equation, I can obtain the discrete Bass model:

$$
\frac{N_{n+1}-N_{n-1}}{2 \delta}=p\left(m-\frac{N_{n+1}+N_{n-1}}{2}\right)+\frac{q}{m}\left(\frac{m}{2}\left(N_{n+1}+N_{n-1}\right)-N_{n+1} N_{n-1}\right) .
$$

The exact solution to equation (23) is written as

$$
N_{n}=m\left(\frac{1-\left(\frac{1-\delta(q+p)}{1+\delta(q+p)}\right)^{\frac{n}{2}}}{1+\frac{q}{p}\left(\frac{1-\delta(q+p)}{1+\delta(q+p)}\right)^{\frac{n}{2}}}\right)
$$

where $n=\frac{t}{\delta}$. The data have to be collected periodically because the time interval is a constant value.

The ceiling $m$ is the same as that of the continuous Bass model and is conserved for any $\delta$ in equation (24), because

$$
N_{n} \rightarrow m \quad \text { as } n \rightarrow \infty .
$$

The ratio of $p$ and $q$ is also the same as that of the continuous Bass model and is conserved for any $\delta$ in equation (24), because $m$ is conserved as shown above and

$$
N_{n} \rightarrow-\frac{m}{\left(\frac{q}{p}\right)} \quad \text { as } n \rightarrow-\infty .
$$

Equation (24) converges equation (3) as follows:

$$
m\left(\frac{1-\left(\frac{1-\delta(q+p)}{1+\delta(q+p)}\right)^{\frac{t}{2 \delta}}}{1+\frac{q}{p}\left(\frac{1-\delta(q+p)}{1+\delta(q+p)}\right)^{\frac{t}{2 \delta}}}\right) \rightarrow m\left(\frac{1-e^{-(q+p) t}}{1+\frac{q}{p} e^{-(p+q) t}}\right) \quad \text { as } \delta \rightarrow 0 .
$$

The difference operator is defined as

$$
\Delta N_{t} \equiv \frac{N_{t+1}-N_{t-1}}{2}
$$

The point of inflection (which is the maximum penetration rate, $\max \left(\Delta N_{t}\right)$ ) occurs when

$$
\bar{n}= \begin{cases}\left\langle n^{*}\right\rangle & \text { (if } \left.\Delta N_{\left\langle n^{*}\right\rangle} \geq \Delta N_{\left\langle n^{*}\right\rangle+1}\right) \\ \left\langle n^{*}\right\rangle+1 & \text { (otherwise) }\end{cases}
$$

where

$$
\begin{aligned}
n^{*} & =2 \frac{\log \frac{p}{q}}{\log \frac{1-\delta(q+p)}{1+\delta(q+p)}}, \\
\left\langle n^{*}\right\rangle & =\left\{n \mid \max \left(n \leq n^{*}\right), n \in \mathbf{Z}\right\} .
\end{aligned}
$$


When $n^{*}$ is an integer,

$$
N_{n^{*}}=m\left(\frac{1}{2}-\frac{p}{2 q}\right)
$$

The above equation is the same as equation (4). Moreover, let

$$
t^{*}=n^{*} \delta
$$

I can show that $t^{*}$ converges the point of inflection in the differential equation as $\delta \rightarrow 0$ as follows:

$$
t^{*}=2 \delta \frac{\log \frac{p}{q}}{\log \frac{1-\delta(q+p)}{1+\delta(q+p)}} \rightarrow-\frac{1}{p+q} \log \left(\frac{p}{q}\right) \quad \text { as } \delta \rightarrow 0 .
$$

The difference between equation (24) and equation (3) is as follows. I expand the following term with $\delta$.

$$
\exp (-2(q+p) \delta)=1-2(q+p) \delta+4(q+p)^{2} \frac{\delta^{2}}{2}+O\left(\delta^{3}\right)+\cdots
$$

Then, I also expand the following term with $\delta$.

$$
\frac{1-\delta(q+p)}{1+\delta(q+p)}=1-2(q+p) \delta+4(q+p)^{2} \frac{\delta^{2}}{2}+O\left(\delta^{3}\right)+\cdots
$$

Equations (35) and (36) show that equation (24) is equivalent to equation (3) until the second order of $\delta$. Therefore, the solution of the difference equation is the same as the solution of the differential equation until the second order of $\delta$.

I compared two difference equations: an ordinary forward difference equation for the Bass model and the difference equation for the discrete Bass model. The parameters were $m=100, p=0.01, q=1.9$, and $\delta=1$, and $N(0)=0.01$ was the initial value. Figures 1 and 2 show the results calculated by the two difference equations. Although Figure 1 shows oscillation, Figure 2 shows that the ceiling is constant.

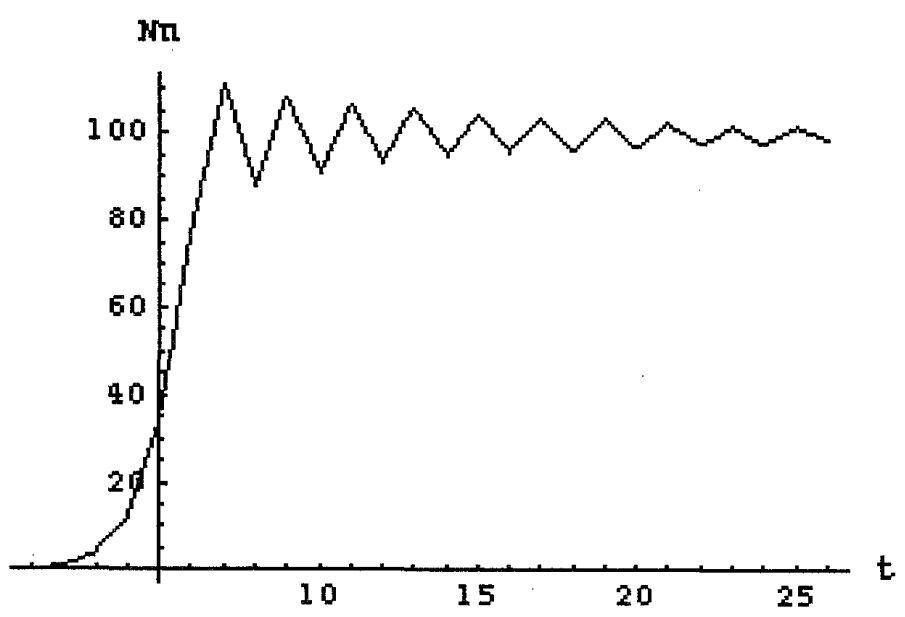

Figure 1: An ordinary forward difference equation for the Bass model.

It is easy to apply OLS to the discrete Bass model because the model is basically a timediscrete equation. The ordinary least squares estimation procedure is the simplest parameter 


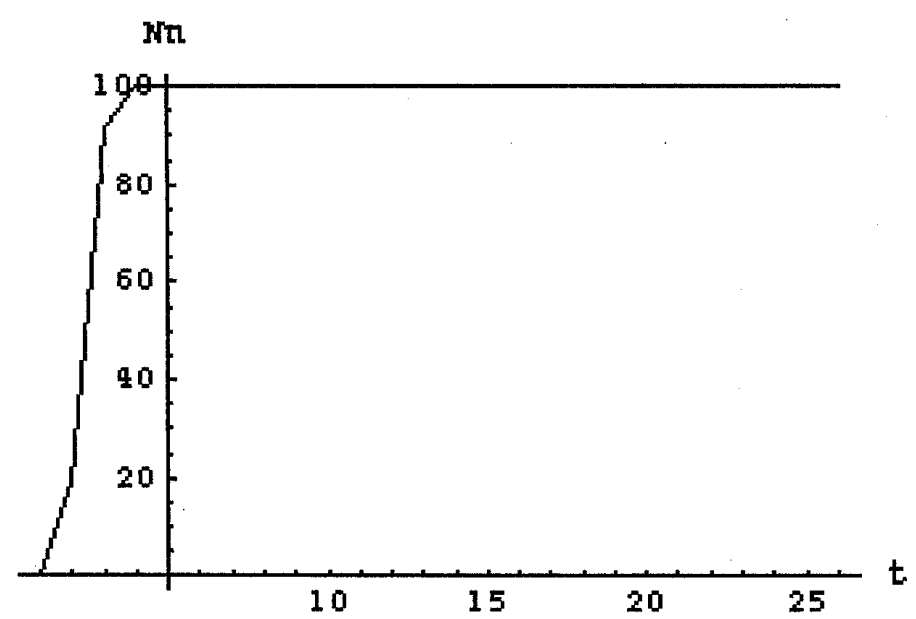

Figure 2: The difference equation for the discrete Bass model.

estimation for the discrete Bass model. In the continuous Bass model, the forward difference equation, which is a regression equation in the OLS procedure, is an approximation of the differential equation. As shown in Figure 1, the approximation of the difference equation is poor. However, in the discrete Bass model, the model itself is directly applied to the regression equation. Moreover, a solution of the discrete Bass model provides the same values as a solution of the continuous Bass model through the following equations:

$$
\begin{aligned}
p_{d} & =k p, \\
q_{d} & =k q, \\
k & =\frac{1}{\delta(p+q)}\left(\frac{1-\exp (-2(q+p))}{1+\exp (-2(q+p))}\right),
\end{aligned}
$$

where $p_{d}$ and $q_{d}$ mean $p$ and $q$ in equation (24), respectively.

I propose two regression models. The first one is the following equation:

$$
S_{n}=2\left(a+b\left(N_{n+1}+N_{n-1}\right)+c N_{n+1} N_{n-1}\right)+\varepsilon(n),
$$

where

$$
\begin{aligned}
S_{n} & =N_{n+1}-N_{n-1}, \\
a & =m p, \\
b & =\frac{q-p}{2}, \\
c & =-\frac{q}{m}, \\
\varepsilon(n) & : \text { error, } E[\varepsilon(n)]=0 .
\end{aligned}
$$

Given regression coefficients ${ }^{2} a, b$, and $c$, parameter estimates $\hat{p}, \hat{q}$, and $\hat{m}$ can be easily obtained as follows:

$$
\begin{aligned}
\hat{p} & =-b+\sqrt{b^{2}-a c} \\
\hat{q} & =b+\sqrt{b^{2}-a c} \\
\hat{m} & =\frac{-b-\sqrt{b^{2}-a c}}{c} .
\end{aligned}
$$

\footnotetext{
${ }^{2} a>0, b>0$, and $c<0$ because $\hat{p}, \hat{q}$, and $\hat{m}$ are positive.
} 
The other regression model is the following equation:

$$
M_{n}=A+B N_{n-1}+C\left(N_{n+1}-N_{n-1}\right)+\varepsilon(n),
$$

where

$$
\begin{aligned}
M_{n} & =N_{n+1} N_{n-1}, \\
A & =\frac{m^{2} p}{q} \\
B & =\frac{m(q-p)}{q}, \\
C & =\frac{m(q-p-1)}{2 q}, \\
\varepsilon(n) & : \operatorname{error}, E[\varepsilon(n)]=0 .
\end{aligned}
$$

Given regression coefficients ${ }^{3} A, B$, and $C$, parameter estimates $\hat{p}, \hat{q}$, and $\hat{m}$ can be easily obtained as follows:

$$
\begin{aligned}
\hat{p} & =\frac{-B+\sqrt{B^{2}+4 A}}{2 B-C}, \\
\hat{q} & =\frac{B+\sqrt{B^{2}+4 A}}{2 B-C} \\
\hat{m} & =\frac{B+\sqrt{B^{2}+4 A}}{2} .
\end{aligned}
$$

These procedures have the advantage of simplicity, which the OLS procedure in the continuous Bass model also offers.

It is also relatively easy to apply the NLS procedure to the discrete Bass model because the discrete Bass model has an exact solution (24). I propose two NLS procedures for the discrete Bass model. One of these provides parameter estimates $\hat{p}, \hat{q}$, and $\hat{m}$ by using the following expressions for the number of adopters $X_{n}$ in the $n$th time interval:

$$
\begin{aligned}
& X_{n}=N_{n+1}-N_{n-1}+\mu_{n} ; \text { or } \\
& X_{n}=m\left(\frac{1-\left(\frac{1-\delta(q+p)}{1+\delta(q+p)}\right)^{\frac{n+1}{2}}}{1+\frac{q}{p}\left(\frac{1-\delta(q+p)}{1+\delta(q+p)}\right)^{\frac{n+1}{2}}}-\frac{1-\left(\frac{1-\delta(q+p)}{1+\delta(q+p)}\right)^{\frac{n-1}{2}}}{1+\frac{q}{p}\left(\frac{1-\delta(q+p)}{1+\delta(q+p)}\right)^{\frac{n-1}{2}}}\right)+\mu_{n}
\end{aligned}
$$

where $\mu_{n}$ is an additive error term.

The other NLS procedure for the discrete Bass model is the following equations:

$$
\begin{aligned}
& Y_{n}=N_{n+1} N_{n}+\nu_{n} \\
& Y_{n}=m^{2}\left(\frac{1-\left(\frac{1-\delta(q+p)}{1+\delta(q+p)}\right)^{\frac{n+1}{2}}}{1+\frac{q}{p}\left(\frac{1-\delta(q+p)}{1+\delta(q+p)}\right)^{\frac{n+1}{2}}}\right)\left(\frac{1-\left(\frac{1-\delta(q+p)}{1+\delta(q+p)}\right)^{\frac{n-1}{2}}}{1+\frac{q}{p}\left(\frac{1-\delta(q+p)}{1+\delta(q+p)}\right)^{\frac{n-1}{2}}}\right)+\nu_{n},
\end{aligned}
$$

where $Y_{n}$ is the ratio between the number of adopters at the $n$th time and that at the $(n+1)$ st time.

\footnotetext{
${ }^{3} A>0, B>0$, and $C<0$ because $\hat{p}, \hat{q}$, and $\hat{m}$ are positive.
} 
These procedures, as well as the NLS procedure for the continuous Bass model, have the advantage that their asymptotic standard errors can be directly estimated. Moreover, since the error term of these procedures may be considered to represent the net effect of sampling errors, excluded variables, and mis-specification of the density function, the derived standard errors for the parameter estimates may be as realistic as those of the NLS procedure for the continuous Bass model.

The OLS procedures of the discrete Bass model overcome the three shortcomings of the OLS procedure in the continuous Bass model: the time-interval bias, standard error, and multicollinearity.

When we use the discrete Bass model to forecast innovation diffusion without a continuoustime Bass model, a time-interval bias does not exist because the model is a discrete model. Furthermore, even if the discrete Bass model is regarded as one procedure to obtain the parameters, these procedures do not suffer from a time-interval bias because a solution of the discrete Bass model gives the same values as a solution of the continuous Bass model as already stated in this section. Therefore, these procedures do not suffer from a time-interval bias.

From equation (23), equation (40) is equivalent to equation (59), and equation (49) is equivalent to equation (61) under no constraints. Therefore, the same parameter estimation is done through both procedures in the discrete Bass model. This is a significant advantage of the discrete Bass model because we can get the global optimum by NLS through OLS. This means both procedures used together overcome the shortcomings of each other separately applied. That is, the standard error of the OLS procedure of the discrete Bass model is obtained through the NLS procedure of the discrete Bass model. Equations (40) and (49) overcome the three shortcomings of NLS: that final parameter estimates are sensitive to the starting values for $p, q$, and $m$, that parameter estimates may sometimes be very slow to converge or may not converge, and that the procedure may not provide a global optimum.

Table 1 shows the condition number, the determinant of correlation matrix $R$, and the variance inflation factors (VIFs) of three procedures: the conventional OLS procedure, the discrete analog 1 of the OLS (40) (dOLS1), and the discrete analog 2 of the OLS (49) (dOLS2), where I chose the exact solution $(p=0.002, q=1, m=100)$ of differential equation (1) as the data from every period from $t=0$ to $t=11$. The VIF in the conventional OLS row is the VIF of the variable $N\left(t_{i-1}\right)$ in equation (8). The value of the VIF of the variable $N\left(t_{i-1}\right)$ is the same as that of the VIF of the other variable $N\left(t_{i}\right)^{2}$ from the definition of the VIF. The VIF in the dOLS1 row is the VIF of the variable $\left(N_{n+1}+N_{n-1}\right)$; the VIF in the dOLS2 row is the VIF of the variables $N_{n-1}$ in Table 1. dOLS2 excludes the problem of multicollinearity. Therefore, a wrong sign for a parameter suggests that the obtained data is not appropriate for the Bass model.

Table 1: Condition number, $\operatorname{det} R$, and VIF.

\begin{tabular}{lccc}
\hline Procedure & Condition number & $\operatorname{det} R$ & VIF \\
\hline Conventional OLS & 14.0111 & 0.01428 & 20.85 \\
dOLS1 & 11.68 & 0.01914 & 12.68 \\
dOLS2 & 3.548 & 0.2059 & 1.000 \\
\hline
\end{tabular}




\section{Parameter Estimation}

The accuracy of the parameter estimation between the conventional OLS procedure and the two OLS procedures in the discrete Bass model was compared. To compare the accuracy of the parameter estimates only, I chose the exact solution $(p=0.002, q=1, m=100)$ of differential equation ( 1 ) as the data from every period from $t=0$ to $t=11$ (the same data as used in the previous section). This data has a point of inflection when $t^{*}=12.4044074$ and $N\left(t^{*}\right)=49.9$. I analyzed three sets of data; data 1: the data up to just before the point of inflection $(t=0,1, \cdots, 6)$, data 2 : the data up to just after the point of inflection $(t=0,1, \cdots, 7)$, and data 3 : the data until the ceiling $(t=0,1, \cdots, 11)$.

The results of the comparison between the conventional OLS and the proposed OLS procedures in the discrete Bass model are shown in Tables 2,3 , and 4, where $p_{1}$ and $q_{1}$ are the parameters of dOLS1 and $p_{2}$ and $q_{2}$ are the parameters of dOLS2. To compare dOLS1 and dOLS2 to the conventional OLS, $p$ and $q$, which are the parameters of the continuous Bass model, are obtained through the following equations:

$$
\begin{aligned}
p & =\tilde{k} p_{i}, \\
q & =\tilde{k} q_{i}, \\
\tilde{k} & =-\frac{1}{2\left(p_{i}+q_{i}\right)} \log \left(\frac{1-\delta\left(p_{i}+q_{i}\right)}{1+\delta\left(p_{i}+q_{i}\right)}\right), \quad i=1,2 .
\end{aligned}
$$

Table 2: Parameter estimates of the conventional OLS.

\begin{tabular}{ccccc}
\hline & $p$ & $q$ & $q / p$ & $m$ \\
\hline data 1 & 0.00734 & 1.61 & 218.9 & 55.71 \\
data 2 & 0.00981 & 1.41 & 144.1 & 71.61 \\
data 3 & 0.0225 & 0.961 & 42.63 & 97.27 \\
\hline
\end{tabular}

Table 3: Parameter estimates of the dOLS1.

\begin{tabular}{ccccccc}
\hline & $p$ & $q$ & $p_{1}$ & $q_{1}$ & $q_{1} / p_{1}$ & $m$ \\
\hline data 1 & 0.002 & 1 & 0.00152 & 0.761 & 500 & 100 \\
data 2 & 0.002 & 1 & 0.00152 & 0.761 & 500 & 100 \\
data 3 & 0.002 & 1 & 0.00152 & 0.761 & 500 & 100 \\
\hline
\end{tabular}

Table 4: Parameter estimates of the dOLS2.

\begin{tabular}{lcccccc}
\hline & $p$ & $q$ & $p_{2}$ & $q_{2}$ & $q_{2} / p_{2}$ & $m$ \\
\hline data 1 & 0.002 & 1 & 0.00152 & 0.761 & 500 & 100 \\
data 2 & 0.002 & 1 & 0.00152 & 0.761 & 500 & 100 \\
data 3 & 0.002 & 1 & 0.00152 & 0.761 & 500 & 100 \\
\hline
\end{tabular}

Tables 5 and 6 show the accuracy of the OLS procedures in the discrete Bass model: dOLS1 and dOLS2. Both OLS procedures in the discrete Bass model provide accurate parameter estimates in the continuous Bass model. Because I used the exact solution 
as the data, an accurate procedure would reproduce the values of the parameters in the exact solution. Tables 3 and 4 show that both OLS procedures in the discrete Bass model reproduced $m, p$, and $q$ perfectly, even though the data did not include the point of inflection and there were fewer than eight data points.

Table 5: Accuracy of parameter estimates in dOLS1.

\begin{tabular}{lcccc}
\hline & $|p-0.002|$ & $|q-1|$ & $\left|q_{1} / p_{1}-500\right|$ & $|m-100|$ \\
\hline data 1 & $3.990 \mathrm{E}-17$ & $5.329 \mathrm{E}-15$ & $1.262 \mathrm{E}-11$ & $1.378 \mathrm{E}-12$ \\
data 2 & $3.166 \mathrm{E}-17$ & $3.331 \mathrm{E}-15$ & $6.253 \mathrm{E}-12$ & $3.268 \mathrm{E}-13$ \\
data 3 & $8.973 \mathrm{E}-16$ & $8.327 \mathrm{E}-15$ & $2.285 \mathrm{E}-10$ & $1.279 \mathrm{E}-13$ \\
\hline
\end{tabular}

Table 6: Accuracy of parameter estimates in dOLS2.

\begin{tabular}{lcccc}
\hline & $|p-0.002|$ & $|q-1|$ & $\left|q_{2} / p_{2}-500\right|$ & $|m-100|$ \\
\hline data 1 & $6.072 \mathrm{E}-18$ & $2.220 \mathrm{E}-15$ & $3.411 \mathrm{E}-13$ & $7.248 \mathrm{E}-13$ \\
data 2 & $1.431 \mathrm{E}-17$ & $6.661 \mathrm{E}-16$ & $3.865 \mathrm{E}-12$ & $1.137 \mathrm{E}-13$ \\
data 3 & $2.64545 \mathrm{E}-17$ & less than 1.0E-18 & $6.480 \mathrm{E}-12$ & less than 1.0E-18 \\
\hline
\end{tabular}

The accuracy was also estimated from the ratio of the two parameters because the ratio of the two parameters of the discrete model is conserved in any time interval $\delta$. The conventional OLS procedure has poor accuracy despite using the exact solution of the differential equation as the data. In particular, the conventional OLS procedure yields poor estimates of the parameters with data 1 , which has seven data points not including the point of inflection. This is consistent with the findings of Heeler and Hustad [8] and Srinivasan and Mason [23]. Through empirical studies, they found that stable and robust estimates for the parameters of the basic diffusion models cannot be obtained unless one uses at least eight data points including the point of inflection. The estimates of the parameters with data 2 were also not accurate enough, even though data 2 satisfies the condition of at least eight data points including the point of inflection.

Whenever a data set is a set of an exact solution of equation (1), the dOLS1 and dOLS2 procedures completely reproduce values of the parameters, e.g., $m, p$, and $q$; theoretically, this is because the solution of equation (23) is the same as that of equation (1) through equations (62), (63), and (64). It is independent of the number of data points or the values of the parameters. However, the conventional OLS procedure does not reproduce values of the parameters and depends on the number of data points as shown in Table (2) because regression equation (8) does not have an exact solution and gives only an approximation of the Bass model.

Moreover, regression equation (8) of the conventional OLS procedure can perfectly fit data that are far from the exact solution of the Bass model. For example, I prepared the data of Table 7 as data that were far from the exact solution of the Bass model, which were illustrated in Figure 3. As shown in Table 8, regression equation (8) fitted the data perfectly even though the data of Table 7 cannot be actually observed. On the other hand, the dOLS1 and dOLS2 procedures both provide a worse fit in terms of the mean absolute deviation and the mean squared error, as shown in Table 8, than does OLS; here, the error term of dOLS2 was translated as equation (65),

$$
\varepsilon(n)=\frac{q}{m} \varepsilon(n) .
$$




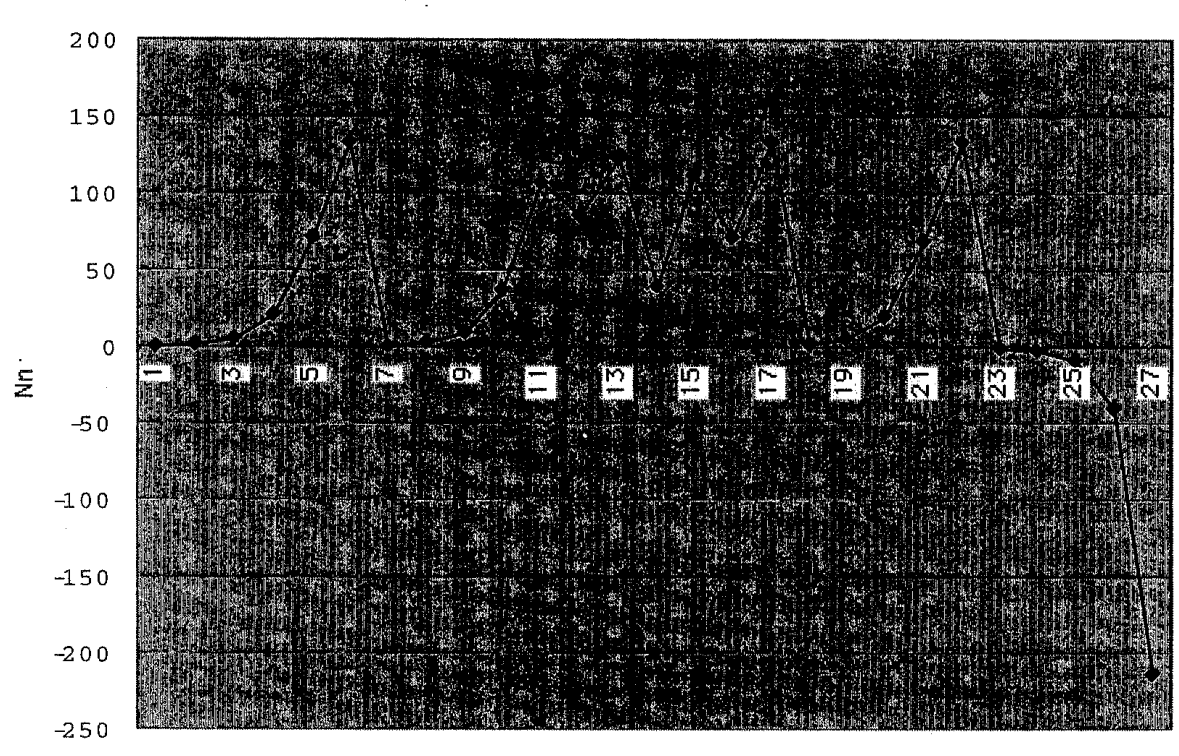

$\mathrm{n}$

Figure 3: Data far from the exact solution of the Bass model.

Furthermore, the dOLS1 and dOLS2 procedures yielded the wrong sign of parameter $p$ for the same data set as Table 9 . As discussed at the end of this section, the wrong sign of the parameter provided by the procedures of the discrete Bass model suggested that the data of Table 7 were not appropriate for the Bass model.

Table 7: Data far from the exact solution of the Bass model.

\begin{tabular}{rrrrrr}
\hline$n$ & \multicolumn{1}{c}{ Data } & $n$ & \multicolumn{1}{c}{ Data } & $n$ & \multicolumn{1}{c}{ Data } \\
\hline 1 & 0.01 & 11 & 106.4349777 & 21 & 69.01715426 \\
2 & 1.039897 & 12 & 85.82342663 & 22 & 133.477418 \\
3 & 5.116747457 & 13 & 122.4656555 & 23 & -0.911735793 \\
4 & 20.63038922 & 14 & 39.70286212 & 24 & -2.66276368 \\
5 & 70.5468642 & 15 & 112.124902 & 25 & -9.837136395 \\
6 & 133.1761867 & 16 & 71.21854939 & 26 & -41.15325179 \\
7 & 0.296083795 & 17 & 132.9995587 & 27 & -214.0091786 \\
8 & 2.178744373 & 18 & 1.001760586 & 28 & -2226.894479 \\
9 & 9.550782237 & 19 & 4.96691901 & 29 & -157656.0796 \\
10 & 36.37109789 & 20 & 20.07789832 & & \\
\hline
\end{tabular}

Table 8: Fit statistics for the three estimation procedures for the Bass model.

\begin{tabular}{lcc}
\hline Procedure & Mean Absolute Deviation & Mean Squared Error \\
\hline OLS & $5.81404 \mathrm{E}-11$ & $5.34 \mathrm{E}-21$ \\
dOLS1 & 71.7211507 & 6928.76843 \\
dOLS2 & 57.4644679 & 6255.08989 \\
\hline
\end{tabular}

I also evaluated the discrete Bass model by using actual diffusion data. This data was the same as that used by Mahajan et al.[13], which was diffusion data for seven products: 
Table 9: Parameter estimates for the three estimation procedures for the Bass model.

\begin{tabular}{cccc}
\hline Procedure & $p$ & $q$ & $m$ \\
\hline OLS & 0.01 & 3 & 100 \\
dOLS1 & -0.004693221 & 1.493157342 & 14717.17075 \\
dOLS2 & -0.007577906 & 0.843251539 & 6663.316838 \\
\hline
\end{tabular}

room air conditioners, color televisions, clothes dryers, ultrasound, mammography, foreign language, and accelerated program. These seven products represent a diversity of innovations and data types for which a minimum of eight annual data points, including the peak (point of inflection), are available. In addition, these products have been used extensively in the diffusion modeling literature to illustrate the application of alternative diffusion models or estimation procedures $[2,12,21,23]$.

To compare the predictive performance of the four estimation procedures, the OLS and the NLS procedure in the continuous Bass model and the two OLS procedures in the discrete Bass model, results related to fit statistics are given in Table 10 . The numbers $(1,2, \cdots, 7)$ in the left column represent, respectively, room air conditioners, color televisions, clothes dryers, ultrasound, mammography, foreign language, and accelerated program. The fit statistics of dOLS2 cannot be compared with those of the other estimation procedures directly because the error term of dOLS2 is different from the error terms of the other estimation procedures. However, from equations (40) and (49), the error term $\varepsilon(n)$ is regarded as

$$
\varepsilon(n)=\frac{m}{q} \varepsilon(n)
$$

Therefore, I compared the fit statistics of dOLS2 with those of other procedures by using this equation.

Results related to the parameter estimates are given in Tables 11, 12, and 13, where the parameter estimates of dOLS1 and dOLS2 in Tables 12 and 13 show the values of $p$ and $q$ in equations (62) and (63) for comparison with other procedures. The parameter estimates of dOLS1 and dOLS2 are the same as those of the corresponding NLS procedures as stated in the previous section.

Table 10: Fit statistics for the four estimation procedures for the Bass model using all available data.

\begin{tabular}{ccccccccc}
\hline & \multicolumn{3}{c}{ Mean Absolute Deviation } & \multicolumn{4}{c}{ Mean Squared Error } \\
\cline { 2 - 5 } & OLS & dOLS1 & dOLS2 & OLS & NLS & dOLS1 & dOLS2 \\
\hline 1 & 173.2 & 144.6 & 92.7 & 97.2 & 41,265 & 26,267 & 13,205 & 15,177 \\
2 & 392.4 & 276.8 & 188.2 & 194.6 & 282,522 & 119,474 & 38,477 & 40,320 \\
3 & 111.8 & 101.5 & 65.0 & 74.1 & 20,818 & 16,367 & 7,692 & 9,115 \\
4 & $\beta$ & 3.0 & 1.96 & 2.21 & $\beta$ & 11.6 & 5.26 & 6.09 \\
5 & $\beta$ & 1.7 & 1.1 & 1.1 & $\beta$ & 3.9 & 2.19 & 2.30 \\
6 & $\beta$ & 0.7 & 0.23 & 0.24 & $\beta$ & 0.5 & 0.0949 & 0.0993 \\
7 & 2.2 & 1.9 & 0.65 & 0.68 & 11.3 & 6.2 & 0.528 & 0.544 \\
\hline
\end{tabular}

Of the four procedures (the OLS, MLE, NLS, and AE procedures in the continuous Bass model), the NLS procedure provides the best fit to the data [13]. Mahajan et al. state that 
Table 11: Parameter estimates of $m$ for the four estimation procedures for the Bass model and the discrete Bass model using all available data.

\begin{tabular}{lcccc}
\hline Product & OLS & NLS & dOLS1 & dOLS2 \\
\hline Room air conditioners & $17.1 \mathrm{E} 6$ & $18.7 \mathrm{E} 6$ & $18.0 \mathrm{E} 6$ & $17.1 \mathrm{E} 6$ \\
Color televisions & $35.5 \mathrm{E} 6$ & $39.7 \mathrm{E} 6$ & $39.1 \mathrm{E} 6$ & $38.4 \mathrm{E} 6$ \\
Clothes dryers & $15.3 \mathrm{E} 6$ & $16.5 \mathrm{E} 6$ & $16.19 \mathrm{E} 6$ & $15.3 \mathrm{E} 6$ \\
Ultrasound & $\beta$ & 167.4 & 187.2 & 180.2 \\
Mammography & $\beta$ & 111.4 & 122.1 & 121.2 \\
Foreign language & $\beta$ & 37.6 & 40.1 & 39.6 \\
Accelerated program & 63.6 & 64.4 & 65.5 & 65.1 \\
\hline
\end{tabular}

Table 12: Parameter estimates of $p$ for the four estimation procedures for the Bass model and the discrete Bass model using all available data.

\begin{tabular}{lcccc}
\hline Product & OLS & NLS & dOLS1 & dOLS2 \\
\hline Room air conditioners & 0.0170 & 0.0094 & 0.0139 & 0.0107 \\
Color televisions & 0.0357 & 0.0185 & 0.02448 & 0.02194 \\
Clothes dryers & 0.0196 & 0.0136 & 0.01790 & 0.014322 \\
Ultrasound & $\beta$ & 0.0013 & -0.01755 & -0.02826 \\
Mammography & $\beta$ & 0.0004 & -0.02501 & -0.030308 \\
Foreign language & $\beta$ & 0.0019 & -0.0249 & -0.02871 \\
Accelerated program & 0.0120 & 0.0007 & -0.01825 & -0.0215363 \\
\hline
\end{tabular}

Table 13: Parameter estimates of $q$ for the four estimation procedures for the Bass model and the discrete Bass model using all available data.

\begin{tabular}{lcccc}
\hline Product & OLS & NLS & dOLS1 & dOLS2 \\
\hline Room air conditioners & 0.4049 & 0.3748 & 0.3842 & 0.42412 \\
Color televisions & 0.6719 & 0.6159 & 0.6162 & 0.64012 \\
Clothes dryers & 0.3481 & 0.3267 & 0.3229 & 0.363769 \\
Ultrasound & $\beta$ & 0.6204 & 0.5537 & 0.63077 \\
Mammography & $\beta$ & 0.8606 & 0.7734 & 0.81747 \\
Foreign language & $\beta$ & 0.6968 & 0.6961 & 0.72534 \\
Accelerated program & 0.8476 & 0.9283 & 0.9597 & 0.99695 \\
\hline
\end{tabular}

assuming global optimum parameter estimates, the NLS procedure should, by definition, provide the best fit in terms of the mean squared error [13]. However, a comparison of the fit statistics in Table 10 indicates that both dOLS1 and dOLS2 provided a better fit to the data than did the OLS or NLS in terms of the mean absolute deviation and mean squared error. The fit statistics of dOLS1 were the best of all. A $\beta$ in Table 10 shows that the OLS procedure yielded an incorrect sign for the regression coefficient $\hat{\alpha}_{1}$ in the regression equation.

Tables 11, 12, and 13 show the estimated parameters of the OLS, NLS, dOLS1, and dOLS2 procedures. Again, $\beta$ shows that the OLS procedure yielded an incorrect sign for the regression coefficient $\hat{\alpha}_{1}$ in the regression equation. The results for the parameter estimates summarized in Table 12 indicate that both dOLS1 and dOLS2 provide the wrong sign for 
the regression coefficient $a$ in equation (40) and for the regression coefficient $A$ in equation (49) for ultrasound, mammography, foreign language, and accelerated program. Both $a$ in equation (40) and $A$ in equation (49) are the regression coefficients of the constant term.

The wrong sign in Table 12, however, does not indicate multicollinearity. Tables 14,15, and 16, respectively, show the condition number, the determinant of the correlation matrix, and the variance inflation factors for each product. These tables show that multicollinearity does not exist in dOLS2. The products that have the wrong signs have smaller condition numbers, larger determinants of the correlation matrices, and smaller VIFs than the products that have the right signs. Therefore, the wrong sign of a parameter suggests that the obtained data is not appropriate for the Bass model.

Table 14: Condition number.

\begin{tabular}{lccc}
\hline Product & OLS & dOLS1 & dOLS2 \\
\hline Room air conditioners & 11.943 & 12.615 & 7.743 \\
Color televisions & 13.321 & 15.768 & 10.123 \\
Clothes dryers & 13.145 & 14.499 & 9.723 \\
Ultrasound & 13.380 & 13.436 & 4.513 \\
Mammography & 14.982 & 13.648 & 3.703 \\
Foreign language & 13.132 & 13.213 & 4.700 \\
Accelerated program & 13.546 & 11.736 & 3.503 \\
\hline
\end{tabular}

Table 15: Determinant of correlation matrix.

\begin{tabular}{lccc}
\hline Product & OLS & dOLS1 & dOLS2 \\
\hline Room air conditioners & 0.01913 & 0.01614 & 0.03135 \\
Color televisions & 0.01453 & 0.009096 & 0.01152 \\
Clothes dryers & 0.01485 & 0.01138 & 0.01817 \\
Ultrasound & 0.01565 & 0.01459 & 0.08556 \\
Mammography & 0.01222 & 0.01383 & 0.1650 \\
Foreign language & 0.01658 & 0.01518 & 0.08084 \\
Accelerated program & 0.01578 & 0.01973 & 0.1836 \\
\hline
\end{tabular}

Table 16: Variance inflation factors.

\begin{tabular}{lccc}
\hline Product & OLS & dOLS1 & dOLS2 \\
\hline Room air conditioners & 14.003 & 13.577 & 2.323 \\
Color televisions & 15.537 & 15.432 & 1.785 \\
Clothes dryers & 15.021 & 15.498 & 2.202 \\
Ultrasound & 17.52 & 15.488 & 1.36 \\
Mammography & 22.121 & 16.19 & 1.013 \\
Foreign language & 17.525 & 15.129 & 1.505 \\
Accelerated program & 20.189 & 13.256 & 1.048 \\
\hline
\end{tabular}




\section{Conclusion}

The discrete Bass model is described with a difference equation that has an exact solution. The exact solution is equivalent to the exact solution of the differential equation that describes the Bass model when the time interval approaches 0 . The exact solution of the discrete Bass model is equivalent to that of the conventional Bass model up to the square of the time interval. Therefore, the exact solution of the discrete Bass model gives a very good approximation of the solution of the conventional Bass model when the time interval is sufficiently small. The ceiling $m$ and the ratio $q / p$ is conserved for any time interval. Moreover, when the transformation to $p$ and $q$ is done, a solution of the discrete Bass model provides the same values as a solution of the continuous Bass model. The discrete Bass model enables us to analyze the diffusion process with only the discrete model because the discrete Bass model has an exact solution and the solution provides the same values as a solution of the continuous Bass model.

When the exact solution is used as the input data, the parameter estimation procedures in the discrete Bass model always reproduce the values of the parameters perfectly. It is independent of the number of data points or the values of the parameters. The OLS and the NLS in the discrete Bass model give the same parameter estimates under no constraints. Although the regression equation of the conventional OLS procedure could perfectly fit data far from the exact solution of the Bass model, the dOLS1 and dOLS2 procedures indicated that such data were not appropriate for the Bass model. For the actual data used by Mahajan et al., both the dOLS1 and the dOLS2 procedures provided a better fit to the data than did the OLS or the NLS procedure in terms of mean absolute deviation and mean squared error. The parameter estimation procedures in the discrete Bass model are superior to the conventional procedures in terms of these two criteria. The two criteria determine the superiority of the parameter estimations in models, such as the Bass model, which have exact solutions.

The parameter estimation procedures in the discrete Bass model have certain advantages compared to those of the conventional Bass model. The OLS procedures of the discrete Bass model overcome the three shortcomings of the OLS procedure in the continuous Bass model: the time-interval bias, standard error, and multicollinearity. Though the wrong signs of the parameters have been regarded as a problem caused by multicollinearity, I found that the wrong signs could be used to judge whether the Bass model works for data.

In the discrete Bass model and in the OLS for a continuous Bass model, the data must be collected periodically because the time interval is constant. The discrete Bass model can be applied if the data is translated into data in the longest interval. If the data is not collected for a constant interval and the data should not be translated into data in the longest interval, a new discrete Bass model whose time interval is not constant has to be derived.

The meanings of parameters $p$ and $q$ are defined through a hazard function. The meanings, e.g., 'innovators' and 'imitators', have played an important role in the Bass model. However, a direct relation between the discrete Bass model and the hazard function has yet to be discovered. Therefore, further studies are needed to determine the direct relation between the discrete Bass model and the hazard function, and to give meanings to parameters $p$ and $q$ through the hazard function.

The approach taken in this paper can be applied to other models if a discrete equation that has an exact solution is derived. For example, Satoh [20] has proposed a discrete Gompertz curve model and the parameter estimation. 


\section{Acknowledgment}

I am grateful to Professor Tohru Ozaki at the Institute of Statistical Mathematics for his stimulating discussions.

\section{References}

[1] J. Arndt: Role of product-related conversations in the diffusion of a new product. Journal of Marketing Research, 4 (1967) 291-295.

[2] F.M. Bass: A new product growth model for consumer durables. Management Science, 15 (1969) 215-227.

[3] I. Bernhardt and K.M. MacKenzie: Some problems in using diffusion models for new products. Management Science, 19 (1972) 187-200.

[4] L. Von Bertalanffy: Quantitative laws in metabolism and growth. Quarterly Review Biology, 32 (1957) 217-231.

[5] R. Chatterjee and J. Eliashberg: The innovation diffusion process in a heterogeneous population: A micromodeling approach. Management Science, 36 (1990) 1057-1079.

[6] L.A. Fourt and J.W. Woodlock: Early prediction of market success for grocery products. Journal of Marketing, 25 (1960) 31-38.

[7] R.E. Frank, W.F. Massy and T.S. Robertson: The determinants of innovative behavior with respect to a branded, frequently purchased food product. In L.G. Smith (ed.): Proceedings of the American Marketing Association (American Marketing Association, Chicago, 1963), 312-323.

[8] R.M. Heeler and T.P. Hustad: Problems in predicting new product growth for consumer durables. Management Science, 26 (1980) 1007-1020.

[9] R. Hirota: Nonlinear partial difference equations. V. Nonlinear equations reducible to linear equations. Journal of the Physical Society of Japan, 46 (1979) 312-319.

[10] D. Jain and R.C. Rao: Effect of price on the demand for durables. Journal of Business and Economic Statistics, 8 (1990) 163-170.

[11] C.W. King, Jr: Fashion adoption: A rebuttal to the 'trickle down' theory. In S.A. Greyser (ed.): Proceedings of the American Marketing Association (American Marketing Association, Chicago, 1963), 108-125.

[12] S.B. Lawton and W.H. Lawton: An autocatalytic model for the diffusion of educational innovations. Educational Administration Quarterly, 15 (1979) 19-53.

[13] V. Mahajan, C.H. Mason and V. Srinivasan: An evaluation of estimation procedures for new product diffusion models. In V. Mahajan and Y. Wind (eds.): Innovation Diffusion Models of New Product Acceptance (Ballinger Cambridge, Massachusetts, 1986), 203232.

[14] V. Mahajan, E. Muller and R.K. Srivastava: Using innovation diffusion models to develop adopter categories. Journal of Marketing Research, 27 (1990) 37-50.

[15] V. Mahajan and S. Sharma: A simple algebraic estimation procedure for innovation diffusion models of new product acceptance. Technological Forecasting and Social Change, 30 (1986) 331-346.

[16] V. Mahajan and Y. Wind: Innovation diffusion models of new product acceptance: A reexamination. In V. Mahajan and Y. Wind (eds.): Innovation Diffusion Models of New Preduct Acceptance (Ballinger Cambridge, Massachusetts, 1986), 3-25.

[17] E. Mansfield: Technical change and the rate of imitation. Econometrica, 29 (1961) $741-766$. 
[18] S.S. Oren and R.G. Schwartz: Diffusion of new products in risk-sensitive markets. Journal of Forecasting, 7 (Oct.-Dec., 1988) 273-287.

[19] T.S. Rebertson: Determinants of innovative behavior. In R. Moyer (ed.) : Proceedings of the American Marketing Association (American Marketing Association, Chicago, 1967), 328-332.

[20] D. Satoh: A discrete Gompertz equation and a software reliability growth model. IEICE Trans. Inf. \& Syst., E83-D (2000) 1508-1513.

[21] D. Schmittlein and V. Mahajan: Maximum likelihood estimation for an innovation diffusion model of new product acceptance. Marketing Science, 1 (1982) 57-78.

[22] A.J. Silk: Overlap among self-designated opinion leaders: A study of selected dental products and services. Journal of Marketing Research, 3 (1966) 255-259.

[23] V. Srinivasan and C.H. Mason: Nonlinear least squares estimation of new product diffusion models. Marketing Science, 5 (1986) 169-178.

[24] S.M. Tanny and N.A. Derzko: Innovators and imitators in innovation diffusion modeling. Journal of Forecasting, 7 (Oct.-Dec., 1988) 225-231.

[25] M. Yamada and R. Furukawa: A classification of diffusion patterns of new products (in Japanese). Marketing Science, 4 (1995) 16-36.

Daisuke Satoh

NTT Service Integration Laboratories 3-9-11 Midori-cho Musashino-shi

Tokyo 180-8585 Japan

E-mail: satoh.daisuke@lab.ntt.co.jp 